\title{
APTITUD FÍSICA RELACIONADA CON SALUD EN NIÑOS ESCOLARIZADOS DE 8 A 12 AÑOS DE UNA INSTITUCIÓN PÚBLICA DE BUCARAMANGA
}

\author{
${ }^{1}$ Claudia Lucía Ariza-García, ${ }^{2}$ Javier Parra-Patiño, ${ }^{3}$ Alejandra Valentina Otárola-Veas, \\ ${ }^{4}$ Linda Victoria Suárez-Rodríguez, ${ }^{5}$ Adriana Angarita-Fonseca. \\ ${ }^{1}$ Magíster en Pedagogía. Docente del programa de Fisioterapia de la Universidad de Santander - UDES. Colombia. \\ ${ }^{2}$ Especialista en Medicina Aplicada a la Actividad Física y el deporte. Docente Escuela de Fisioterapia de la Universidad Industrial de Santander, UIS. \\ ${ }^{3}$ Ingeniero en Estadística. Instituto Nacional de Estadística de Chile. \\ ${ }_{4}^{4}$ Estudiante de Fisioterapia. Universidad de Santander, Bucaramanga. Colombia. \\ ${ }^{5}$ Magíster en Epidemiología. Docente del Programa de Fisioterapia de la Universidad de Santander, UDES. Colombia.
}

Autor responsable de correspondencia: Adriana Angarita Fonseca Correo electrónico: adriangarita@udes.edu.co

\section{RESUMEN}

Objetivos: determinar el nivel de AFRS y explorar la asociación entre capacidad aeróbica, flexibilidad y fuerza muscular con el exceso de peso en escolares de 8 a 12 años.

Materiales y métodos: se realizó un estudio de corte transversal, se seleccionaron por conveniencia los grados cuarto y quinto del Instituto Tecnológico Salesiano Eloy Valenzuela. La prevalencia de sobrepeso y obesidad se determinó según la OMS-CDC y el FITNESSGRAM. La AFRS se evaluó mediante la batería FITNESSGRAM. Se aplicaron modelos de regresión de Poisson simples y múltiples para calcular las razones de prevalencia (RP) y sus intervalos de confianza del 95\% (IC95\%).

Resultados: participaron 66 escolares (10,4 \pm 0,82 años), 19,6\% mujeres y 48,5\% de grado cuarto. El 78,8\% y 39,4\% estaba por debajo de la zona de aptitud fisica saludable en las cualidades fuerza muscular en extensores de tronco y capacidad aeróbica, respectivamente. La prevalencia de sobrepeso y obesidad según el criterio OMS-CDC fue del 30,3\%. Las cualidades físicas asociadas con el sobrepeso y obesidad fueron capacidad aeróbica (RP 2,32 IC95\% 1,08-4,96) y la fuerza muscular de miembros superiores (RP 3,53 IC95\% 1,28-9,69). Conclusiones: el porcentaje de niños por debajo de la zona saludable fue mayor en las cualidades fisicas, fuerza muscular y capacidad aeróbica. Las cuales se asociaron con exceso de peso. Esto es especialmente relevante dado que una adecuada capacidad aeróbica en la niñez se ha relacionado con un perfil cardiometabólico más saludable. [Ariza-García CL, Parra-Patiño J, Otálora-Veas AV, SuárezRodríguez LV, Angarita-Fonseca A. Aptitud física relacionada con salud en niños escolarizados de 8 a 12 años en una institución pública de Bucaramanga. Ustasalud 2015;14:32-39].

Palabras clave: Aptitud física; índice de masa corporal, fuerza muscular, niño.

\section{FITNESS-RELATED HEALTH OF SCHOOL CHILDREN 8-12 YEARS OF A PUBLIC SCHOOL FROM BUCARAMANGA}

\begin{abstract}
Objectives: to determine the level of RPF and to explore the association among aerobic fitness, flexibility and muscle strength with overweight and obesity in schoolchildren 8-12 years.

Methods: a cross-sectional study was performed; we selected for convenience the fourth and fifth degrees from "Instituto Tecnologico Salesiano Eloy Valenzuela". The prevalence of overweight and obesity was determined according to the WHO-CDC and the FITNESSGRAM. The HRPF was assessed using the FITNESSGRAM battery. Simple and multiple Poisson regression models were applied to calculate prevalence ratios (PR) and their confidence intervals 95\% (CI 95\%).

Results: a total of 66 students (10.4 \pm 0.82 years), $19.6 \%$ women, and $48.5 \%$ of the fourth grade, were included. $78.8 \%$ and $39.4 \%$ were below the healthy fitness zone in the trunk extension strength and the aerobic fitness qualities, respectively. The prevalence of overweight and obesity according to WHO-CDC criteria was $30.3 \%$. The physical qualities associated with overweight and obesity were aerobic fitness (PR 2.32 CI 95\% 1.08 to 4.96) and muscle strength of upper limbs (PR CI 95\% 1.28 to 9.69 3.53).

Conclusions: the percentage of children below the healthy area was higher in the muscular strength and aerobic fitness physical qualities. Which were associated with overweight and obesity. This is especially relevant given that adequate aerobic fitness in childhood has been linked to a healthier cardio-metabolic profile.
\end{abstract}

Keywords: Physical fitness; body mass index; muscle strength; child.

Recibido para publicación: 13 de octubre de 2014. Aprobado para publicación: 23 de noviembre de 2014. 


\section{INTRODUCCIÓN}

La Aptitud Física Relacionada con Salud (AFRS) se centra en el alcance de objetivos que contribuyen al adecuado funcionamiento orgánico, se enfoca en el bienestar del individuo y concede importancia a las cualidades, composición corporal, capacidad aeróbica, fuerza, resistencia y flexibilidad muscu$\operatorname{lar}^{1}$. Su relación con la obesidad ha sido estudiada por varios autores. Se ha encontrado una asociación negativa entre el Índice de Masa Corporal (IMC) y la AFRS, especialmente con bajos niveles de resistencia muscular $^{2,3,}$ capacidad aeróbica ${ }^{4,5}$, flexibilidad ${ }^{6}$ y actividad física ${ }^{7}$. Esta relación se explica, en parte, por el aumento en la dedicación del tiempo de niños y jóvenes a actividades de ocio, como ver ver televisión, usar la computadora y videojuegos que afecta un estilo de vida activo ${ }^{8,9}$.

Igualmente, la relación de la AFRS con las enfermedades cardiovasculares la ubican como un importante predictor de morbilidad y mortalidad por estas causas ${ }^{10-13}$. Además, la evidencia científica ha demostrado que estas enfermedades no se originan en la adultez; por el contrario, el nivel de AFRS y los factores de riesgo cardiovascular en la edad adulta están directamente relacionados con la AFRS que se obtuvo en la infancia o adolescencia ${ }^{14-16}$. Lo anterior, sumado a que la valoración de la AFRS permite identificar y controlar los factores que pueden generar alteraciones orgánicas; su evaluación en las escuelas y colegios se convierte en una necesidad sentida que serviría de base para plantear programas y proyectos que fomenten una adecuada AFRS que disminuya el riesgo de presentar obesidad y enfermedades crónicas no transmisibles como las cardiovasculares en la adultez.

Se han propuesto diferentes baterías para evaluar la AFRS en niños y adolescentes, la mayoría con valores de referencia para población norteamericana, aunque también existen para población de Europa, China y Australia ${ }^{17,18}$. Se destacan las baterías Physical Best, la National Children and Youth Fitness Study planteada por el Council of Physical Fitness and Sport, la EUROFIT y la batería FITNESSGRAM; esta última tiene como objetivo aumentar la conciencia de los padres sobre el estado físico de los niños; para ello, se determina si el evaluado está en la Zona de Condición Física Saludable (ZFS) con base en estándares de rendimiento físico previamente definidos, esta zona corresponde al nivel desde el cual es posible prevenir los trastornos y deficiencias que resultan de una vida sedentaria ${ }^{19}$.

En Colombia, Tovar-Mojica y colaboradores (2008) relacionaron los niveles de AFRS y actividad física con el sobrepeso en una población de varones entre
7 y 18 años20; por otro lado, Jáuregui y Ordóñez (2003) estandarizaron las pruebas de aptitud fisica relacionadas con el desempeño físico deportivo (AFRD), evaluaron la composición corporal, flexibilidad, capacidad aeróbica, velocidad, potencia muscular, fuerza muscular, tiempo de reacción y coordinación; sin embargo, dentro de la metodología no está explícito el número de participantes entre 7 y 16 años que fueron evaluados ${ }^{21}$. En otro estudio, Correa (2008) determinó el perfil antropométrico y la AFRD de varones futbolistas entre 7 y 16 años de Bogotá ${ }^{22}$. Por su parte, Villera y Petro (2010) describieron la AFRD en niños y niñas de 10 a 12 años de instituciones educativas de Montería ${ }^{23}$. En el ámbito local, Cohen y colaboradores (2014) evaluaron la relación entre la fuerza muscular, capacidad aeróbica y el perfil cardio-metabólico en 669 escolares de 8 a 14 años de un colegio público de Bucaramanga $^{24}$. Es evidente la escasa publicación de artículos científicos sobre la AFRS en escolares colombianos, por esta razón se propone determinar la AFRS mediante la aplicación de la batería de prueba FITNESSGRAM en niños escolarizados de 8 a 12 años de una institución pública de Bucaramanga y establecer la asociación entre las cualidades físicas y el exceso de peso.

\section{MATERIALES Y MÉTODOS}

Se realizó un estudio de corte transversal. La muestra fue seleccionada por conveniencia y estuvo conformada por 66 de 73 estudiantes de cuarto y quinto grado de la sede $C$ del Instituto Tecnológico Salesiano Eloy Valenzuela, un colegio público del área urbana de la ciudad de Bucaramanga, Colombia. Se incluyeron los niños y niñas entre los 8 y 12 años de edad que aceptaron participar voluntariamente en el estudio, se excluyeron los participantes que presentaron alguna discapacidad física, trastorno metabólico, enfermedades cardiovasculares, neuromusculares, respiratorias o cualquier alteración que pudiera complicar la condición de salud al realizar las pruebas.

La recolección de la información la hicieron en octubre de 2005 cuatro estudiantes de último grado de Fisioterapia previamente entrenados, quienes estandarizaron el procedimiento al realizar una prueba piloto con 20 estudiantes de tercer grado del mismo Instituto y de las mismas edades. Las pruebas se realizaron en las instalaciones del colegio, a excepción de la prueba de la Milla, que se desarrolló en la cancha deportiva más cercana a la Institución, durante los días miércoles, jueves y viernes de tres semanas consecutivas en la jornada de clases (7:00 a.m. a 12:00 m.). El tiempo de duración de la batería fue de aproximadamente 30 minutos por participante. Se midieron las variables sociodemográficas 
edad, género y grado escolar y cuatro cualidades físicas: composición corporal mediante el índice de masa corporal, la capacidad aeróbica con la prueba de la milla corriendo, la resistencia muscular aplicando las pruebas flexión de tronco, extensión de tronco y flexión de brazos, y la flexibilidad muscular con las pruebas flexión del tronco hacia delante desde la posición sedente y la prueba de extensión de hombros.

El día previo al trabajo de campo los participantes fueron notificados sobre la realización de las pruebas. Durante la evaluación, inicialmente se registraron los datos pertenecientes a las variables sociodemográficas y posteriormente se realizaron las pruebas siguiendo el protocolo del FITNESSGRAM $^{19}$ en el siguiente orden:

Composición corporal: la masa corporal se estableció utilizando una balanza digital con una precisión antropométrica de $100 \mathrm{~g}$ y con una capacidad máxima de $150 \mathrm{Kg}$ que era calibrada diariamente. La talla se obtuvo a través de un tallímetro portátil con una base horizontal en madera y una reglilla en milímetros ubicada a $90^{\circ}$ de la base. Estas mediciones se obtuvieron con los participantes en posición de pie, sin zapatos y con la mirada dirigida sobre la horizontal. Posteriormente, se determinó el índice de masa corporal (IMC) que relaciona la masa corporal en $\mathrm{Kg}$ dividida por la estatura en metros elevada al cuadrado. La prevalencia de sobrepeso y obesidad se determinó según dos criterios: el criterio OMS-CDC, en el cual se considera en sobrepeso y obesidad el participante por encima del percentil 85, según datos estandarizados para Colombia y el criterio FITNESSGRAM, el cual considera exceso de grasa por encima del $10 \%$ al $20 \%$ en varones y del $15 \%$ al $25 \%$ en mujeres.

Capacidad aeróbica: en la prueba de la milla corriendo el participante recorrió esta distancia lo más rápido posible al ritmo de sus capacidades y dado el caso les fue permitido caminar. La distancia se demarcó ubicando conos en los extremos del área rectangular de la cancha de cemento, en las rectas largas y en las rectas cortas. La prueba se realizó en subgrupos de 4 estudiantes; a cada niño se le asignó un número para facilitar el registro de las vueltas completadas y determinar el tiempo total gastado en la realización de la prueba.

Flexibilidad muscular: en la prueba flexión del tronco hacia delante desde la posición sedente se empleó un cajón de $60 \mathrm{~cm}$ de largo, $27 \mathrm{~cm}$ de ancho y $30,5 \mathrm{~cm}$ de alto con una regla en la parte superior haciendo coincidir la pulgada 9 con el apoyo de la planta del pie.
El estudiante descalzo acomodó las caderas al frente de la caja para alinear el cuerpo, posteriormente, ubicó los pies en la superficie horizontal de la caja, extendió completamente una de las piernas con la base del pie contra la caja, mientras la otra rodilla fue flexionada con la planta del pie apoyada en el piso y separada de la otra a una distancia de 2-3 pulgadas. Los brazos fueron extendidos hacia adelante sobre la escala de medición con las dos manos hacia abajo, colocadas una encima de la otra, la flexión de tronco se realizó con la espalda recta y la cabeza mirando al frente cuatro veces con ambas manos a lo largo de la escala, manteniendo la posición del cuarto intento por lo menos 1 segundo. Este mismo procedimiento se realizó para la otra pierna y se registró el desplazamiento en pulgadas.

En la prueba de extensión de hombros el participante llevaba un hombro en máxima flexión y rotación externa, flexionaba el codo de modo que la mano quedara con la palma en contacto con la espalda mientras que el otro hombro se encontraba en extensión y rotación interna con el dorso de la mano en contacto con la espalda. Se registró si el participante alcanzaba a tocarse los dedos en esta posición o no. El procedimiento se realizó en ambos miembros superiores.

Resistencia muscular: en la prueba flexión de tronco el participante partió de la posición supina sobre una colchoneta, con las rodillas flexionadas a un ángulo de $140^{\circ}$, plantas de los pies apoyadas en la colchoneta con las piernas ligeramente separadas, brazos estirados y paralelos al tronco con las palmas de las manos y cabeza en contacto con la colchoneta. Los dedos de las manos estuvieron separados del pulgar y sus pulpejos quedaron en el ribete o inicio de la banda ubicada debajo de las piernas. La prueba terminaba cuando el estudiante no continuaba más o cuando completaba las 75 flexiones a una cadencia determinada. Se registró el número de repeticiones realizadas.

En la prueba de extensión de tronco la posición inicial fue en decúbito prono sobre la colchoneta. Los pies en plantiflexión y las manos situadas debajo de los muslos. Los estudiantes realizaron extensión de tronco de manera muy suave y controlada, a una altura máxima de 12 pulgadas $(30.48 \mathrm{~cm})$. Se conservó un alineamiento neutral de la cabeza con la espina dorsal. El evaluador situó la regla en el piso enfrente del estudiante y determinó la distancia del piso a la barbilla de este en pulgadas.

El participante que realizó la prueba de flexión de brazos adoptó la posición prona sobre la colchoneta apoyado sobre las manos, las cuales estuvieron alineadas con los hombros; mientras que, los dedos 
y las piernas estuvieron extendidos y separados. El estudiante flexionó y luego estiró los brazos y piernas, se supervisó la alineación de la espalda con la cabeza y los dedos de los pies. El cuerpo bajó hasta que los hombros lograron un ángulo de $90^{\circ}$ y los brazos estuvieron paralelos al piso. Este movimiento fue repetido tanto como fue posible y se registró el número de repeticiones.

La clasificación de las cualidades físicas de los participantes se hizo teniendo en cuenta la zona de aptitud fisica saludable para cada niño evaluado con base en los estándares de aptitud física del FITNESSGRAM.

\section{Análisis estadístico}

La información de cada participante se digitó por duplicado, por personas diferentes, en bases de datos independientes en Excel. Se confrontaron las dos bases de datos en el subprograma Validate de Epi Info 6.04d y se identificaron y corrigieron errores de digitación. El análisis se realizó en el programa estadístico Stata Versión 9.1. Se describieron las características sociodemográficas y las cualidades físicas de la población de estudio aplicando medidas de tendencia central y dispersión o porcentajes y sus respectivos intervalos de confianza del 95\% (IC95\%) según la naturaleza y distribución de las variables. La evaluación de las posibles asociaciones entre las cualidades físicas, edad, género y grado escolar con el sobrepeso y obesidad se realizó mediante el modelo de regresión simple y múltiple de Poisson para calcular las Razones de Prevalencia (RP) y sus IC95\%. Se consideró un nivel de significancia alpha $=0,05$.

\section{Consideraciones éticas}

Este estudio se consideró de riesgo mínimo, según la Resolución 008430 de 1993 del Ministerio de Protección Social de Colombia y fue aprobado por el Comité de Ética de la Universidad de Santander. Se solicitó la participación voluntaria a los estudiantes y la autorización de sus padres mediante consentimiento informado escrito.

\section{RESULTADOS}

Participaron en este estudio 66 estudiantes, 53 varones y 13 mujeres con edades comprendidas entre los 9 y 12 años $(10,4 \pm 0,82)$, el $48,5 \%$ cursaba cuarto grado de primaria y el restante $51,5 \%$ quinto grado. Los resultados de las pruebas que evalúan las diferentes cualidades físicas mostraron algunas diferencias estadísticamente significativas por edad; la tendencia observada es que a mayor edad aumenta el número de repeticiones de la prueba de la fuerza muscular de los abdominales y disminuye el tiempo gastado en realizar la prueba de la milla; mientras que, en la prueba de los rotadores de hombro derecho se observó menor flexibilidad en los participantes de 9 años de edad comparado con las otras edades (Tabla 1).

En relación con el porcentaje de estudiantes que están por debajo de la ZFS (Tabla 2), se encontró que la cualidad fisica menos desarrollada es la fuerza muscular, se destacan la musculatura abdominal y de miembros superiores en los niños de nueve años y la musculatura extensora de tronco en las demás edades. La flexibilidad de miembros inferiores se encontró normal en todos los participantes; mientras que, la flexibilidad de miembros superiores no fue normal principalmente en los niños de nueve años. El porcentaje de participantes que se encontraba por debajo de la ZFS en la prueba de la milla aumentó con la edad, sin llegar a ser estadísticamente significativo dicho aumento. En cuanto a la composición corporal, el porcentaje de niños con un IMC por debajo de la ZFS fue bajo.

La prevalencia de sobrepeso y obesidad global y por género, según los criterios FITNESSGRAM y OMSCDC se muestra en la Tabla 3. Los resultados revelan diferencias entre los dos criterios, siendo mayor la prevalencia encontrada con el criterio OMS-CDC. Por género se encontró mayor prevalencia de sobrepeso $\mathrm{y}$ obesidad en los varones.

Las razones de prevalencia crudas y ajustadas se presentan en la Tabla 4, en el análisis bivariado se encontró una asociación estadísticamente significativa solo con la capacidad aeróbica ( $\mathrm{RP}=2,85$ IC95\% $1,31-6,20)$ que se mantuvo en el análisis multivariado (RP 2,32 IC95\% 1,08-4,96). Adicionalmente, en el análisis multivariado se encontró asociación con la fuerza de miembros superiores (RP 3,53 IC95\% 1,28-9,69).

\section{DISCUSIÓN}

Los principales hallazgos de este estudio incluyen un elevado porcentaje de escolares con exceso de peso, clasificación en zona no saludable según el FITNESSGRAM principalmente en las pruebas de fuerza muscular y capacidad aeróbica. A su vez se encontró asociación significativa entre fuerza muscular de miembros superiores y capacidad aeróbica por debajo de la zona saludable con exceso de peso.

La prevalencia de sobrepeso y obesidad, según los criterios OMS-CDC en el presente estudio (30,3\% IC95\% 19,6-42,9\%), estuvo por encima de la reportada por la Encuesta Nacional de Salud y Nutrición de 2010, bajo el criterio OMS, la prevalencia de sobrepeso y obesidad nacional en población de 5 a 17 años fue de 17,5\% y en Santander de 18,8\%25. 
Tabla 1. Resultados de las pruebas del FITNESSGRAM en la población de niños de 8-12 años de un colegio público de Bucaramanga-2005, según edad

\begin{tabular}{|c|c|c|c|c|c|}
\hline \multirow[b]{2}{*}{ Cualidad física } & \multicolumn{5}{|c|}{ Edad (años) } \\
\hline & $\begin{array}{c}9 \\
\text { Prom } \pm \mathrm{DE}\end{array}$ & $\begin{array}{c}10 \\
\text { Prom } \pm \mathrm{DE}\end{array}$ & $\begin{array}{c}11 \\
\text { Prom } \pm \mathrm{DE}\end{array}$ & $\begin{array}{c}12 \\
\text { Prom } \pm \mathrm{DE}\end{array}$ & $\mathbf{p}$ \\
\hline \multicolumn{6}{|l|}{ Composición corporal } \\
\hline Peso (kg) & $31,6 \pm 10,1$ & $30,9 w \pm 6,6$ & $34,9 \pm 7,1$ & $37,2 \pm 7,3$ & 0,527 \\
\hline Estatura (cm) & $132,6 \pm 7,2$ & $135,4 \pm 6,6$ & $139,9 \pm 7,1$ & $142,8 \pm 7,4$ & 0,979 \\
\hline $\mathrm{IMC}\left(\mathrm{kg} / \mathrm{m}^{2}\right)$ & $17,7 \pm 4,2$ & $16,7 \pm 2,4$ & $17,7 \pm 2,7$ & $18,1 \pm 2,1$ & 0,205 \\
\hline \multicolumn{6}{|l|}{ Capacidad aeróbica } \\
\hline Tiempo milla (min) & $11,2 \pm 3,1$ & $11,0 \pm 1,6$ & $10,4 \pm 3,4$ & $10,5 \pm 1,8$ & 0,002 \\
\hline \multicolumn{6}{|l|}{ Flexibilidad } \\
\hline S\&R derecha $(\mathrm{cm})$ & $32,8 \pm 4,7$ & $33,5 \pm 4,0$ & $31,0 \pm 4,8$ & $34,8 \pm 3,6$ & 0,774 \\
\hline S\&R izquierda $(\mathrm{cm})$ & $32,9 \pm 5,3$ & $32,2 \pm 4,3$ & $30,9 \pm 4,9$ & $34,9 \pm 4,2$ & 0,848 \\
\hline Rotación hombro derecho (cm) & $-2,6 \pm 7,1$ & $3,0 \pm 2,7$ & $1,58 \pm 5,1$ & $2,6 \pm 3,1$ & 0,001 \\
\hline Rotación hombro izquierdo (cm) & $-2,9 \pm 3,2$ & $0,00 \pm 4,2$ & $-1,8 \pm 5,2$ & $-0,5 \pm 3,8$ & 0,484 \\
\hline \multicolumn{6}{|l|}{ Fuerza muscular } \\
\hline $\begin{array}{l}\text { Abdominales } \\
\text { (No. de repeticiones) }\end{array}$ & $7,3 \pm 11,4$ & $18,4 \pm 16,3$ & $26,5 \pm 26,2$ & $24,3 \pm 9,6$ & 0,006 \\
\hline Extensión de tronco (cm) & $14,8 \pm 3,3$ & $17,1 \pm 4,3$ & $17,0 \pm 4,2$ & $16,4 \pm 2,7$ & 0,579 \\
\hline $\begin{array}{l}\text { Flexiones de pecho } \\
\text { (No. de repeticiones) }\end{array}$ & $7,1 \pm 7,2$ & $7,1 \pm 6,8$ & $5,9 \pm 6,1$ & $4,2 \pm 3,1$ & 0,318 \\
\hline
\end{tabular}

Prom: Promedio. DE: Desviación estándar. IMC: Índice de masa corporal. S\&R: Sit and Reach.

Tabla 2. Distribución de los participantes que se encuentran por debajo de la zona de condición física saludable (ZFS) según el FITNESSGRAM, por edad. Población de niños de 8-12 años de un colegio público de Bucaramanga-2005

\begin{tabular}{|c|c|c|c|c|c|c|c|c|c|c|c|c|c|c|c|}
\hline \multirow{3}{*}{ Cualidad física } & \multicolumn{12}{|c|}{ Edad } & \multirow{2}{*}{\multicolumn{3}{|c|}{$\begin{array}{l}\text { Global } \\
(n=66)\end{array}$}} \\
\hline & \multicolumn{3}{|c|}{$9(n=8)$} & \multicolumn{3}{|c|}{$10(n=27)$} & \multicolumn{3}{|c|}{$11(n=25)$} & \multicolumn{3}{|c|}{$12(n=6)$} & & & \\
\hline & $\mathbf{n}$ & $\%$ & IC95\% & $\mathbf{n}$ & $\%$ & IC95\% & $\mathbf{n}$ & $\%$ & IC95\% & $\mathbf{n}$ & $\%$ & IC95\% & $\mathbf{n}$ & $\%$ & IC95\% \\
\hline \multicolumn{16}{|l|}{ Composición corporal } \\
\hline IMC & 0 & 0,0 & - & 2 & 7,4 & $0,9-24,3$ & 1 & 4,0 & $0,1-20,4$ & 0 & 0,0 & - & 3 & 4,6 & $1,0-12,7$ \\
\hline \multicolumn{16}{|l|}{ Capacidad aeróbica } \\
\hline Milla & 3 & 37,5 & $8,5-75,5$ & 9 & 33,3 & $16,5-53,9$ & 11 & 44,0 & $24,4-65,1$ & 3 & 50,0 & $11,8-88,2$ & 26 & 39,4 & $27,6-52,2$ \\
\hline \multicolumn{16}{|l|}{ Flexibilidad } \\
\hline S\&R & 0 & 0,0 & - & 0 & 0,0 & - & 0 & 0,0 & - & 0 & 0,0 & - & 0 & 0,0 & \\
\hline Rotación Hombro & 5 & 62,5 & $24,5-91,5$ & 8 & 29,6 & $13,8-50,2$ & 10 & 40,0 & $21,1-61,3$ & 2 & 33,3 & $4,3-77,7$ & 25 & 37,9 & $26,2-50,7$ \\
\hline \multicolumn{16}{|l|}{ Fuerza muscular } \\
\hline Abdominales & 6 & 75,0 & $34,9-96,8$ & 11 & 40,7 & $22,4-61,2$ & 9 & 36,0 & $17,9-57,5$ & 1 & 16,7 & $0,4-64,1$ & 27 & 40,9 & $29,0-53,7$ \\
\hline Extensión de tronco & 4 & 50,0 & $15,7-84,3$ & 18 & 66,7 & $46,0-83,5$ & 24 & 96 & $79,7-99,9$ & 6 & 100 & $54,1-100$ & 52 & 78,8 & $67,0-87,9$ \\
\hline Flexiones de pecho & 5 & 62,6 & $24,5-91,5$ & 13 & 48,2 & $28,7-68,1$ & 17 & 68,0 & $46,5-85,1$ & 5 & 83,3 & $35,9-99,6$ & 40 & 60,6 & $47,8-72,4$ \\
\hline
\end{tabular}

IMC: Índice de masa corporal. IC95\%: Intervalo de confianza del 95\%. S\&R: Sit and Reach. 
Tabla 3. Prevalencia de sobrepeso y obesidad por género. Población de niños de 8-12 años de un colegio público de Bucaramanga, 2005

\begin{tabular}{cccccccccc}
\hline \multirow{2}{*}{ Criterio } & \multicolumn{3}{c}{ Femenino } & \multicolumn{3}{c}{ Masculino } & \multicolumn{3}{c}{ Global } \\
\cline { 2 - 10 } & $\mathbf{n}$ & $\%$ & IC95\% & $\mathbf{n}$ & $\mathbf{\%}$ & IC95\% & $\mathbf{n}$ & $\%$ & IC95\% \\
\hline FITNESGRAM & 0 & 0,0 & - & 3 & 5,66 & $1,2-15,7$ & 3 & 4,6 & $0,9-12,7$ \\
OMS-CDC & 3 & 23,1 & $5,0-23,1$ & 17 & 32,1 & $19,9-46,3$ & 20 & 30,3 & $19,6-42,9$ \\
\hline
\end{tabular}

IC95\%: Intervalo de confianza del 95\%.

Tabla 4. Asociación entre las características sociodemográficas evaluadas, capacidad aeróbica, flexibilidad y fuerza muscular con el exceso de peso (Regresión de Poisson Simple y Múltiple). Población de niños de 8-12 años de un colegio público de Bucaramanga-2005

\begin{tabular}{|c|c|c|c|c|c|c|}
\hline \multirow{2}{*}{ Variable } & \multicolumn{2}{|c|}{ Con sobrepeso y obesidad } & \multirow{2}{*}{ RP cruda } & \multirow{2}{*}{ IC95\% } & \multirow{2}{*}{ RP ajustada } & \multirow{2}{*}{ IC95\% } \\
\hline & Sí & No & & & & \\
\hline \multicolumn{7}{|l|}{ Sexo } \\
\hline Masculino & 17 & 36 & 1,39 & $0,47-4,0$ & 1,33 & $0,54-3,32$ \\
\hline Femenino & 3 & 10 & Ref. & & & \\
\hline \multicolumn{7}{|l|}{ Grado } \\
\hline Quinto & 12 & 22 & 1,41 & $0,66-3,0$ & 2,34 & $0,77-7,17$ \\
\hline Cuarto & 8 & 24 & Ref. & & & \\
\hline \multicolumn{7}{|l|}{ Edad (años) } \\
\hline 9 y 10 & 10 & 21 & 1,13 & $0,54-2,35$ & 0,89 & $0,40-1,96$ \\
\hline 11 y 12 & 10 & 25 & Ref. & & & \\
\hline \multicolumn{7}{|l|}{ Capacidad aeróbica } \\
\hline Por debajo de la ZFS & 13 & 13 & 2,85 & $1,31-6,20$ & 2,32 & $1,08-4,96$ \\
\hline En o por encima de la ZFS & 7 & 33 & Ref. & & & \\
\hline \multicolumn{7}{|c|}{ Flexibilidad rotadores de hombro } \\
\hline Anormal & 8 & 17 & 1,09 & $0,51-2,29$ & 1,15 & $0,56-2,35$ \\
\hline Normal & 12 & 29 & Ref. & & & \\
\hline \multicolumn{7}{|l|}{ Fuerza de abdominales } \\
\hline Por debajo de la ZFS & 9 & 18 & 1,18 & $0,57-2,47$ & 0,91 & $0,56-2,35$ \\
\hline En o por encima de la ZFS & 11 & 28 & Ref. & & & \\
\hline \multicolumn{7}{|c|}{ Fuerza de extensores de tronco } \\
\hline Por debajo de la ZFS & 14 & 38 & 0,62 & $0,29-1,33$ & 0,36 & $0,10-1,28$ \\
\hline En o por encima de la ZFS & 6 & 8 & Ref. & & & \\
\hline \multicolumn{7}{|c|}{ Fuerza de miembros superiores } \\
\hline Por debajo de la ZFS & 16 & 24 & 2,6 & $0,97-6,9$ & 3,53 & $1,28-9,69$ \\
\hline En la ZFS & 4 & 22 & Ref. & & & \\
\hline
\end{tabular}

ZFS: Zona de Fitness saludable. Ref: Grupo de referencia. RP: Razón de prevalencia. IC95\%: Intervalo de confianza del 95\%.

Aunque los participantes del presente estudio no representan a todos los niños y niñas de 8 a 12 años de Santander, los resultados se acercaron a los reportados por Tovar-Mojica et al. (2008) quienes encontraron una prevalencia de obesidad de $18,1 \%$ y de sobrepeso del 20\%20. También fueron cercanos a los reportados por Cohen et al. (2014), 12,4\% para sobrepeso y $12,9 \%$ para obesidad24. Los resultados indudablemente muestran la necesidad urgente de crear programas o proyectos que fomenten hábitos de vida saludable y disminuyan la obesidad en niños y niñas.

Actualmente hay un interés en establecer la relación entre la condición física, especialmente la capacidad cardiorespiratoria y la fuerza muscular con el estado de salud, incluyendo la obesidad ${ }^{26}$. Bajos niveles de capacidad aeróbica en niños tienden a perpetuarse hasta la vida adulta ${ }^{15,27}$. Así mismo, bajos niveles 
de capacidad aeróbica se han asociado a enfermedades cardio-metabólicas incluyendo intolerancia a la glucosa, hiperlipidemias, arterioesclerosis, entre otras $^{28}$. Adicionalmente, en varios países, bajos niveles de actividad fisica y aptitud fisica se consideran un problema de salud pública.

Los resultados del presente estudio muestran que el $40,9 \%, 78,8 \%, 60,6 \%$ de los escolares se encontraba por debajo de la zona saludable en las pruebas de fuerza muscular de abdominales, extensores de tronco y miembros superiores, respectivamente. Así mismo, el 39,4\% se encontraba por debajo de la zona saludable en la prueba de capacidad aeróbica. Los hallazgos de este estudio concuerdan con otros estudios que reportan que entre el 18 y 30\% de niños está en la zona de bajo riesgo en la prueba de capacidad aeróbica ${ }^{29-31}$. De la misma manera, los hallazgos de este estudio muestran que los niños clasificados por debajo de la zona saludable en la prueba de fuerza de miembros superiores $(\mathrm{RP}=3,53$ IC $95 \% 1,28-9,69)$ y en la prueba de capacidad aeróbica $(\mathrm{RP}=2,32$ IC95\% 1,08-4,96) tienen mayor probabilidad de presentar exceso de peso. Resultados similares se han hallado en otros estudios. Aires et al. (2008) encontraron una relación inversa entre fuerza muscular y capacidad aeróbica e índice de masa corporal en niños y niñas de Portugal ${ }^{32}$. Tokmakidis, Kasambalis y Christodoulos (2006); Huberty, Rosenkranz, Balluff y High (2010); Bustamante y Maia (2013); y Cohen et al. (2014) descubrieron la misma relación en niños de Grecia ${ }^{33}$, Estados Unidos $^{34}$, Perú (35) y Colombia ${ }^{24}$, respectivamente.

Aunque los mecanismo por los cuales la capacidad aeróbica y la fuerza muscular se relacionan con la obesidad no son claros. Se conoce que la obesidad podría aumentar el daño de la fibra muscular durante su ciclo de vida por disminución del contenido de ADN de las células satélite del músculo y disminución de la renovación celular, lo que explica que la potencia y fuerza muscular disminuya ${ }^{36}$. Un estudio mostró un mayor número de triglicéridos y su ubicación central en todos los tipos de fibras musculares en personas obesas en comparación con las no obesas ${ }^{37}$. El exceso de tejido abdominal y en los espacios perimusculares e intramusculares producen altos niveles de proteínas inflamatorias y citoquinas, tales como la proteína C-reactiva, factor de necrosis tumoral (TNF- $\alpha$ ), interleuquina $1 \beta$ y la interleuquina- 6 , entre otros ${ }^{38}$. El ambiente inflamatorio podría estar relacionado con la atrofia muscular y el desarrollo de la sarcopenia ${ }^{39}$.

Por todo lo anterior, las escuelas, las cuales comúnmente administran pruebas de aptitud fisica, son los principales lugares para la identificación de niños de alto riesgo, adicionalmente en este contexto se deberían desarrollar programas o estrategias de prevención eficaces, ya sea para todos los niños o dirigido hacia aquellos con alto riesgo.

Dentro de las limitaciones del estudio se encuentran la selección por conveniencia, por lo cual no se pueden generalizar los resultados de este estudio; la utilización de valores de referencia y puntos de corte calculados para población estadounidense, así como la utilización de pruebas de campo que son mediciones indirectas y pueden llevar a sesgo de información; la naturaleza transversal del estudio que no permite establecer causalidad en la relación fuerza muscular y capacidad aeróbica con exceso de peso.

\section{Financiación}

Programa de Fisioterapia Universidad de Santander.

\section{REFERENCIAS}

1. Caspersen CJ, Powell KE, Christenson GM. Physical activity, exercise and physical fitness: Definitions and distinctions for health-related research. Public Health Rep. 1985;100(2):126-31.

2. Harrell JS, McMurray RG, Bangdiwala SI, Frauman AC, Gansky SA, Bradley CB. Effects of a school-based intervention to reduce cardiovascular disease risk factors in elementary- school children: the Cardiovascular Health in Children (CHIC) study. J Pediatr. 1996;128(6):797-805.

3. Deforche B1, Lefevre J, de Bourdeaudhuij I, Hills AP, Duquet W, Bouckaert J. Physical fitness and physical activity in obese and nonobese Flemish youth. Obes Res. 2003;11(3):434-41.

4. Lloyd LK, Bishop PA, Walker JL, Sharp KR, Richardson MT. The influence of body size and composition on FITNESSGRAM(r) Test performance and the adjustment of FITNESSGRAM(r) Test scores for skinfold thickness in youth. Meas Phys Educ Exerc Sci. 2003;7(4):205-26.

5. Savva SC, Tornaritis MJ, Kolokotroni O, Chadjigeorgiou C, Kourides Y, Karpathios T et al. High cardiorespiratory fitness is inversely associated with incidence of overweight in adolescence: a longitudinal study. Scand J Med Sci Sports. 2014;24(6):982-9.

6. Chen W, Lin CC, Peng CT, Li CI, Wu HC, Chiang J et al. Approaching healthy body mass index norms for children and adolescents from health-related physical fitness. Obes Rev. 2002;3(3):225-32.

7. Lau XC, Chong KH, Poh BK, Ismail MN. Physical activity, fitness and the energy cost of activities: implications for obesity in children and adolescents in the tropics. Adv Food Nutr Res. 2013;70:49-101.

8. Rey-López JP1, Vicente-Rodríguez G, Biosca M, Moreno LA. Sedentary behaviour and obesity development in children and adolescents. Nutr Metab Cardiovasc Dis. 2008;18(3):242-51.

9. LeBlanc AG, Chaput JP, McFarlane A, Colley RC, Thivel D, Biddle SJ, et al. Active video games and health indicators in children and youth: a systematic review. PLoS One. 2013;8(6):e65351.

10. Thomas NE, Baker JS, Davies B. Established and Recently Identified Coronary Heart Disease Risk Factor in Young 
People: the Influence of Physical Activity and Physical Fitness. Sports Med. 2003;33(9):633-50.

11. Ortega FB, Ruiz JR, Castillo MJ, Sjöström M. Physical fitness in childhood and adolescence: a powerful marker of health. Int J Obes (Lond). 2008;32(1):1-11.

12. Ruiz JR, Sui X, Lobelo F, Morrow JR Jr, Jackson AW, Sjöström $\mathrm{M}$ et al. Association between muscular strength and mortality in men: prospective cohort study. BMJ 2008;337:a439.

13. Artero EG, Lee DC, Ruiz JR, Sui X, Ortega FB, Church TS, et al. A prospective study of muscular strength and all-cause mortality in men with hypertension. J Am Coll Cardiol. 2011;57(18):1831-7.

14. Twisk JW1, Kemper HC, van Mechelen W. The relationship between physical fitness and physical activity during adolescence and cardiovascular disease risk factors at adult age. The Amsterdam Growth and Health Longitudinal Study. Int J Sports Med. 2002;23 Suppl 1:S8-14.

15. Trudeau F, Laurencelle L, Shephard RJ. Tracking of physical fitness from childhood to adulthood. Med Sci Sports Exerc. 2004;36(11):1937-43.

16. Rodrigues LP, Leitão R, Lopes VP. Physical fitness predicts adiposity longitudinal changes over childhood and adolescence. J Sci Med Sport. 2013;16(2):118-23.

17. Castro-Piñero J, Artero EG, España-Romero V, Ortega FB, Sjöström M, Suni J et al. Criterion-related validity of fieldbased fitness tests in youth: A systematic review. Br J Sports Med. 2010;44(13):934-43.

18. Cvejić D, Pejović T, Ostojić S. Assessment of physical fitness in children and adolescent. Facta Universitatis. 2013;11(2):135-45.

19. Meredith MD, Welk GJ. FITNESSGRAM/ACTIVITYGRAM. Test Administration Manual. Third edition. Champaign, IL: Human Kinetics Publisher; 2005.

20. Tovar-Mojica G, Gutiérrez-Poveda J, Ibáñez-Pinilla M, Lobelo F. Sobrepeso, inactividad fisica y baja condición física en un colegio de Bogotá, Colombia. Arch Latinoam Nutr. 2008;58(3):265-73.

21. Jáuregui G, Ordóñez ON. Aptitud física: Pruebas estandarizadas en Colombia. Instituto Nacional del Deportes (Coldeportes). Ministerio de Educación Nacional. Bogotá, 2003.

22. Correa JE. Determinación del perfil antropométrico y cualidades físicas de niños futbolistas de Bogotá. Rev Cien Salud Bogotá (Colombia). 2008;6(2):74-84.

23. Villera S, Petro JL. Valoración de la aptitud física de los escolares de 10 a 12 años de Montería, Colombia. Efdeportes. 2010;15(48): Recuperado a partir de: http://www. efdeportes.com/efd148/valoracion-de-la-aptitud-fisicade-los-escolares.htm

24. Cohen DD, Gómez-Arbeláez D, Camacho PA, Pinzón S, Hormiga C, Trejos-Suárez J, et al. Low Muscle Strength Is Associated with Metabolic Risk Factors in Colombian Children: The ACFIES Study. PLoS One. 2014;9(4):e93150.

25. Instituto Colombiano de Bienestar Familiar. Encuesta Nacional de la Situación Nutricional en Colombia 2010 ENSIN. Bogotá: Da Vinci Editores, 2010.

26. García-Artero E, Ortega FB, Ruiz JR, Mesa JL, Delgado M, García M et al. El perfil lipídico-metabólico en los adolescentes está más influido por la condición física que por la actividad fisica (estudio AVENA). Rev Esp Cardiol. 2007;60(6):581-88.
27. Ortega FB, Ruiz JR, Castillo MJ, Moreno L, GonzálezGross M, Warnberg J et al. Bajo nivel de forma física en adolescentes españoles. Importancia para la salud cardiovascular futura (estudio AVENA). Rev Esp Cardiol. 2005;58(8):898-909.

28. Fogelholm M. Physical activity, fitness and fatness: relations to mortality, morbidity and disease risk factors. A systematic review. Obes Rev. 2010;11(3):202-21.

29. Corbin CB, Pangrazi RP. Are American children and youth fit? Res Q Exerc Sport. 1992;63:665-8.

30. Kim J, Must A, Fitzmaurice GM, Gillman MW, Chomitz V, Kramer E et al. Relationship of physical fitness to prevalence and incidence of overweight among schoolchildren. Obes Res. 2005;13(7):1246-54.

31. Mota J, Flores L, Flores L, Ribeiro JC, Santos MP. Relationship of single measures of cardiorespiratory fitness and obesity in young schoolchildren. Am J Hum Biol. 2006;18(3):335-41.

32. Aires L, Silva P, Santos R, Santos P, Ribeiro JC, Mota J. Association of physical fitness and body mass index in youth. Minerva Pediatr. 2008;60(4):397-405.

33. Tokmakidis SP, Kasambalis A, Christodoulos AD. Fitness levels of Greek primary schoolchildren in relationship to overweight and obesity. Eur J Pediatr. 2006;165(12):86774.

34. Huberty JL, Rosenkranz RR, Balluff MA, High R. Describing weight status and fitness in a community sample of children attending after-school programming. J Sports Med Phys Fitness. 2010;50(2):217-28.

35. Bustamante A, Maia J. Weight status and cardiorespiratory fitness in school students in the central region of Peru. Rev. Perú. med. exp. salud pública. 2013;30(3),399407.

36. Peterson JM, Bryner RW, Alway SE. Satellite cells proliferation is reduced in muscles of obese Zucker rats but restored with loading. Am J Physiol Cell Physiol. 2008;295(2):C521-8.

37. Malenfant P, Joanisse DR, Thériault R, Goodpaster BH, Kelley DE, Simoneau JA. Fat content in individual muscle fibers of lean and obese subjects. Int J Obes Relat Metab Disord. 2001;25(9):1316-21.

38. Gregor MF, Hotamisligil GS. Inflammatory mechanisms in obesity. Annu Rev Immunol. 2011;29:415-45.

39. Schaap LA, Pluijm SM, Deeg DJ, Visser M. Inflammatory markers and loss of muscle mass (sarcopenia) and strength. Am J Med. 2006;119(6):526.e9-17.

\section{Correo electrónico de los autores}

Claudia Ariza: claudiaariza15@yahoo.es Javier Parra: jparra@uis.edu.co

Alejandra Valentina Otárola-Veas alejandra.o.veas@gmail.com Linda Victoria Suárez-Rodríguez: lindavicsuarez@gmail.com Adriana Angarita: adriangarita@udes.edu.co 